\title{
An iterative asymptotic expansion method for elliptic eigenvalue problems with oscillating coefficients
}

\author{
Shafigh Mehraeen · Jiun-Shyan Chen · Wei Hu
}

Received: 13 April 2009 / Accepted: 13 October 2009 / Published online: 10 November 2009

(C) The Author(s) 2009. This article is published with open access at Springerlink.com

\begin{abstract}
In this work we propose a method for obtaining fine-scale eigensolution based on the coarse-scale eigensolution in elliptic eigenvalue problems with oscillating coefficient. This is achieved by introducing a 2 -scale asymptotic expansion predictor in conjunction with an iterative corrector. The eigensolution predictor equation is formulated using the weak form of an auxiliary problem. It is shown that large errors exist in the higher eigenmodes when the 2-scale asymptotic expansion is used. The predictor solution is then corrected by the combined inverse iteration and Rayleigh quotient iteration. The numerical examples demonstrate the effectiveness of this approach.
\end{abstract}

Keywords Multi-scale $\cdot$ Asymptotic expansion · Eigenvalue problems $\cdot$ Inverse iteration

\section{Introduction}

Many problems of fundamental and practical importance contain multiple scale features. When the eigenvalue problem involves more than one distinct length scale in its governing equation, single scale numerical solution is ineffective. On the other hand, methods that allow construction of eigensolution of one scale in terms of the other scale remain challenging.

S. Mehraeen · J.-S. Chen $(\bowtie)$

Civil and Environmental Engineering Department, University

of California, Los Angeles (UCLA), 5713 Boelter Hall,

Los Angeles, CA 90095, USA

e-mail: jschen@seas.ucla.edu

W. $\mathrm{Hu}$

Civil and Environmental Engineering Department, University of California, Davis, 2001 Engineering III, Davis, CA 95616, USA
Condensation based methods were among early remedies to resolve the difficulties in multi-scale eigenvalue problems. The main idea of the condensation method is that modal properties of connected subsystems can be reduced to those of a main body retaining the dominant effects of the problem $[14,15]$. An example is a method of dynamic condensation [20] in which the passive coordinates are represented by the active ones, and the eigenvalue problem is solved by a combined technique of Sturm sequence and subspace iteration. In the context of multi-scale analysis of eigenvalue problem, two model reduction methods, operator-function method and quantum scattering analog method, have also been developed $[8-11,26]$. In these approaches, a projection matrix that relates degrees of freedom at different scales is constructed and it enables the solution of macroscopic eigenvalue problem. Multigrid method [21] introduces minimization of the Rayleigh quotient to both global and local grid systems. Multiresolution strategy has been introduced to capture the influence of fine scales on the behavior of the solution on a coarse scale. Multiresolution analysis (MRA) considers the explicit relationship between two adjacent scales and it is not subjected to the restriction of separable length scale $[16,19,29,30]$. MRA has also been applied to model reduction of eigenvalue problems using higher order wavelets [12]. Other methods that consider the interaction between substructure and the full-scale problem have been introduced. An approach proposed for large-scale micromechanical problem considered a solution of the total problem as an expansion of solutions of sub-structural problems with conforming subdomains, where the sub-structural problems are constructed with proper boundary conditions making them mutually separable and relatively inexpensive to solve [31].

Asymptotic analysis for periodic structures has been introduced by Lions, Bensoussan, and Papanicolaou [3]. Asymptotic method has also been introduced for multi-scale 
eigenvalue problems. In an early work by Kesavan $[17,18]$, homogenization of elliptic eigenvalue problems was considered. Based on two-scale asymptotic expansion, leading order equations were derived. Formulations for first and second order corrections for eigenvalues were introduced, and error estimates were obtained based on the correction equations. The approach by Hoog et al. [6] considered a coarse scale solution as an approximation to the fine scale Strum Liouville problems. Albert et al. [1] introduced asymptotic expansion to the field variables of conservation equations, leading to an eigenvalue problem for investigation of the stability of two-layer flow. The homogenized eigenvalue problems have been corrected with the first order correction [22, $27,28]$ as well as higher-order asymptotic expansions $[4,5]$.

Solution of eigenvalue problems has been studied extensively [2]. For large-scale eigenvalue problems, inverse iteration and Rayleigh quotient method have been proposed. The convergence of inverse iteration can be very slow if some eigenvalues are densely distributed, and the combination of inverse iteration and Rayleigh quotient iteration has been shown to achieve cubic rate of convergence [23-25].

This work aims to introduce the asymptotic expansion based method for multi-scale eigenvalue problem. It is shown that the 2-scale asymptotic expansion method does not yield sufficient accuracy in the higher eigenmodes. We consider using the solution of 2-scale asymptotic expansion as the initial guess, and enhance the 2 -scale eigensolution by an inverse iteration and Rayleigh quotient iteration. The outline of this paper is as follows. In Sect. 2, an asymptotic expansion method for eigenvalue problems is introduced as a predictor. The iterative scheme for correcting the 2-scale eigensolution is presented in Sect. 3. Numerical examples are presented in Sect. 4 to demonstrate the effectiveness of the proposed method. Concluding remarks are given in Sect. 5 .

\section{Asymptotic expansion of eigenvalue problems}

Following homogenization theory by Kesavan [17,18], the multi-scale eigenvalue problem and the corresponding leading order equations at different scales are introduced in this section. Let $\Omega$ be a bounded domain in $\mathbb{R}^{n}$ and $\Gamma$ the corresponding boundary. Define $\boldsymbol{x}$ and $\boldsymbol{y}$ as the macroscopic and microscopic coordinates, respectively, and they are related by

$y_{i}=x_{i} / \lambda$

where $\lambda \rightarrow 0$ is the scale ratio. Consider the following differential operator

$L_{\lambda}=-\frac{\partial}{\partial x_{i}}\left(E_{i j}^{\lambda} \frac{\partial}{\partial x_{j}}\right)$ in which we assume the symmetry property of $E_{i j}^{\lambda}$, i.e. $E_{i j}^{\lambda}=$ $E_{j i}^{\lambda}$. In addition, the following assumptions are made: (1) there exists a constant independent of $\lambda, M>0$, such that $\left|E_{i j}^{\lambda}\right| \leq M, 1 \leq i, j \leq n,(2)$ there exists a constant independent of $\lambda, N>0$ such that $\forall \eta_{i}, \eta_{j} \in \mathbb{R}^{n}, E_{i j}^{\lambda} \eta_{i} \eta_{j} \leq$ $N \eta_{i} \eta_{i}, 1 \leq i, j \leq n$.

The strong form of homogeneous Dirichlet eigenvalue problem corresponding to the partial differential operator $L_{\lambda}$ is expressed by

$\left(\mathrm{S}_{\lambda}\right)$ : Find eigenpair $\left(u^{\lambda}, \omega^{\lambda}\right)$ such that

$L_{\lambda} u^{\lambda}=\omega^{\lambda} u^{\lambda} \quad$ in $\Omega$

$u^{\lambda}=0$ on $\Gamma$

The associated weak form of Eq. (2.3) is

$\left(\mathrm{W}_{\lambda}\right)$ : Find eigenpair $\left(u^{\lambda}, \omega^{\lambda}\right) \in H_{0}^{1}(\Omega) \times \mathbb{R}$ such that $\forall v \in H_{0}^{1}(\Omega)$

$a_{\lambda}\left(u^{\lambda}, v\right)=\omega^{\lambda}\left(u^{\lambda}, v\right)$

where

$a_{\lambda}\left(u^{\lambda}, v\right)=\int_{\Omega} E_{i j}^{\lambda} \frac{\partial u^{\lambda}}{\partial x_{i}} \frac{\partial v}{\partial x_{j}} d \Omega$
$\left(u^{\lambda}, v\right)=\int_{\Omega} u^{\lambda} v d \Omega$

The solution of the eigenvalue problem in Eq. (2.3) by replacing the differential operator with homogenized operator $L_{0}$ approaches the homogeneous solution of the original multiscale eigenvalue problem. The homogenized eigenvalue problem can be stated as

(S): Find eigenpair $\left(u^{[0]}, \omega^{[0]}\right)$ such that

$L_{0} u^{[0]}=\omega^{[0]} u^{[0]}$ in $\Omega$

$u^{[0]}=0 \quad$ on $\Gamma$

where

$L_{0}=-\frac{\partial}{\partial x_{i}}\left(E_{i j}^{[0]} \frac{\partial}{\partial x_{j}}\right)$

in which constant $E_{i j}^{[0]}$ are the homogenized coefficients. The weak form of Eq. (2.7) reads

(W) : Find eigenpair $\left(u^{[0]}, \omega^{[0]}\right) \in H_{0}^{1}(\Omega) \times \mathbb{R}$

such that $\forall v \in H_{0}^{1}(\Omega)$

$a\left(u^{[0]}, v\right)=\omega^{[0]}\left(u^{[0]}, v\right)$ 
where

$$
\begin{aligned}
& a\left(u^{[0]}, v\right)=\int_{\Omega} E_{i j}^{0} \frac{\partial u^{[0]}}{\partial x_{i}} \frac{\partial v}{\partial x_{j}} d \Omega \\
& \left(u^{[0]}, v\right)=\int_{\Omega} u^{[0]} v d \Omega
\end{aligned}
$$

In order to separate the coarse- and fine-scale components of eigenvalues and eigenfunctions, asymptotic expansion is introduced. Consider the following asymptotic expansion:

$$
\begin{aligned}
\omega^{\lambda}=\omega^{[0]}+ & \lambda \omega^{[1]}+\lambda^{2} \omega^{[2]}+O\left(\lambda^{3}\right) \\
u^{\lambda}(\boldsymbol{x}, \boldsymbol{y})= & u^{[0]}(\boldsymbol{x}, \boldsymbol{y})+\lambda u^{[1]}(\boldsymbol{x}, \boldsymbol{y}) \\
& +\lambda^{2} u^{[2]}(\boldsymbol{x}, \boldsymbol{y})+O\left(\lambda^{3}\right)
\end{aligned}
$$

We have $\omega^{\lambda} \rightarrow \omega^{[0]}$ and $u^{\lambda} \rightarrow u^{[0]}$ as $\lambda \rightarrow 0$. Let $\omega^{[0]}$ and $u^{[0]}$ be any eigenvalue and eigenfunction of the homogenized problem (S) in Eq. (2.7), respectively. Consider the following auxiliary problem

$$
\begin{aligned}
& L_{\lambda} w^{\lambda}=\omega^{[0]} u^{[0]} \text { in } \Omega \\
& w^{\lambda}=0 \text { on } \Gamma
\end{aligned}
$$

The corresponding weak form is to find $w^{\lambda} \in H_{0}^{1}(\Omega)$, $\forall v \in H_{0}^{1}(\Omega)$,

$a_{\lambda}\left(w^{\lambda}, v\right)=\omega^{[0]}\left(u^{[0]}, v\right)$

Using $u^{\lambda}$ and $w^{\lambda}$ as test functions in Eqs. (2.14) and (2.4), respectively, and comparing the resultant Eq. (2.14) with Eq. (2.4), we have

$\omega^{\lambda}\left(u^{\lambda}, w^{\lambda}\right)=\omega^{[0]}\left(u^{[0]}, u^{\lambda}\right)$

where the symmetry of $a_{\lambda}$ (., .) has been employed. Equation (2.15) is a predictor equation whose solution $w^{\lambda}$ is employed as an initial guess. Here we assumed that $u^{\lambda}$ and $w^{\lambda}$ have the same coarse component $u^{[0]}$ such that $w^{\lambda} \rightarrow u^{\lambda}$ as $\lambda \rightarrow 0$, thus

$$
\begin{aligned}
w^{\lambda}(\boldsymbol{x}, \boldsymbol{y})= & u^{[0]}(\boldsymbol{x}, \boldsymbol{y})+\lambda w^{[1]}(\boldsymbol{x}, \boldsymbol{y})+\lambda^{2} w^{[2]}(\boldsymbol{x}, \boldsymbol{y}) \\
& +O\left(\lambda^{3}\right)
\end{aligned}
$$

One can normalize an eigenfunction such that

$$
\left(u^{\lambda}, u^{\lambda}\right)=1
$$

Introducing asymptotic expansion to $u^{\lambda}$ in Eq. (2.17) leads to the following conditions

$$
\left(u^{[0]}, u^{[0]}\right)=1, \quad\left(u^{[0]}, u^{[1]}\right)=0
$$

Substituting Eqs. (2.16)-(2.18) into the predictor equation (2.15) gives the leading order equations from which fine-scale components of eigenvalues can be approximated

$$
\begin{aligned}
O\left(\lambda^{0}\right): \omega^{[0]}= & \omega^{[0]} \\
O\left(\lambda^{1}\right): \omega^{[1]}= & -\omega^{[0]}\left(u^{[0]}, w^{[1]}\right) \\
O\left(\lambda^{2}\right): \omega^{[2]}= & -\omega^{[0]}\left\{\left(u^{[0]}, w^{[2]}\right)+\left(u^{[1]}, w^{[1]}\right)\right\} \\
& -\omega^{[1]}\left(u^{[0]}, w^{[1]}\right) \\
\approx & -\omega^{[0]}\left\{\left(u^{[0]}, w^{[2]}\right)+\left(w^{[1]}, w^{[1]}\right)\right\} \\
& -\omega^{[1]}\left(u^{[0]}, w^{[1]}\right)
\end{aligned}
$$

where it is assumed that $u^{[1]}$ can be approximated by $w^{[1]}$. Thus, upon solving for functions $w^{[1]}$ and $w^{[2]}$, fine-scale components of eigenvalues, $\omega^{[1]}$ and $\omega^{[2]}$, can be obtained from above equations.

In order to solve for $w^{[1]}$ and $w^{[2]}$, one can employ the strong form of the auxiliary problem in Eq. (2.13). First, consider the following chain rule

$$
\begin{aligned}
\partial(.) / \partial x_{i} & =\partial(.) /\left.\partial x_{i}\right|_{y}+\left(\partial(.) /\left.\partial y_{i}\right|_{x}\right)\left(\partial y_{i} / \partial x_{i}\right) \\
& =\partial(.) /\left.\partial x_{i}\right|_{y}+\lambda^{-1}\left(\partial(.) /\left.\partial y_{i}\right|_{x}\right)
\end{aligned}
$$

Employing above chain rule in Eq. (2.13) leads to the decoupled differential operator as

$$
\begin{aligned}
L_{\lambda}(.)= & \lambda^{-2} L_{[-2]}(.)+\lambda^{-1} L_{[-1]}(.)+\lambda^{0} L_{[0]}(.) \\
& +O(\lambda)
\end{aligned}
$$

where

$$
\begin{aligned}
L_{[-2]}(.)= & -\partial / \partial y_{i}\left(E_{i j}^{\lambda}(\boldsymbol{x}) \partial(.) / \partial y_{j}\right) \\
L_{[-1]}(.)= & -E_{i j}^{\lambda}(\boldsymbol{x}) \partial^{2}(.) / \partial x_{i} \partial y_{j}-\partial / \partial y_{i} \\
& \times\left(E_{i j}^{\lambda}(\boldsymbol{x}) \partial(.) / \partial x_{j}\right) \\
L_{[0]}(.)= & -E_{i j}^{\lambda}(\boldsymbol{x}) \partial^{2}(.) / \partial x_{i} \partial x_{j}
\end{aligned}
$$

Substituting Eqs. (2.21) and (2.22) into Eq. (2.13) yields the following leading order equations

$$
\begin{aligned}
& O\left(\lambda^{-2}\right)=0: u^{[0]}(\boldsymbol{x}, \boldsymbol{y})=u^{[0]}(\boldsymbol{x}) \\
& O\left(\lambda^{-1}\right)=0: L_{[-2]} \alpha_{k}=L_{[-2]} y_{k}
\end{aligned}
$$

$O\left(\lambda^{0}\right)=0: L_{[0]} u^{[0]}+L_{[-1]} w^{[1]}+L_{[-2]} w^{[2]}$

$$
=\omega^{[0]} u^{[0]} \text { in } \Omega
$$

$u^{[0]}=0$ on $\Gamma$

where $\alpha_{j}$ is a coupling function between coarse- and finescale components of an eigenfunction [3] such that

$w^{[1]}(x, y)=-\alpha_{k}(y)\left(\partial u^{[0]} / \partial x_{k}\right)$ 
The coupling function $\alpha_{k}$ can be solved from Eq. (2.24). Substituting the scale coupling relation (Eq. 2.26) into the last leading order equation $O\left(\lambda^{0}\right)=0$ (Eq. 2.25 and neglecting $w^{[2]}$, we obtain the following homogenized eigenvalue problem

$L_{0} u^{[0]}=\omega^{[0]} u^{[0]}$ in $\Omega$

$u^{[0]}=0$ on $\Gamma$

where

$E_{i j}^{[0]}=1 /|Y| \int_{\Omega_{Y}} E_{i k}^{\lambda}(\boldsymbol{x})\left(\delta_{j k}-\frac{\partial \alpha_{j}}{\partial y_{k}}\right) d \Omega$

in which $|Y|$ is the area (volume) of the unit cell with domain $\Omega_{Y}$. One can proceed further to solve for $w^{[2]}$ by

$w^{[2]}(\boldsymbol{x}, \boldsymbol{y})=\beta_{i j}(\boldsymbol{y})\left(\partial^{2} u^{[0]} / \partial x_{i} \partial x_{j}\right)$

where $\beta_{i j}(\boldsymbol{y})$ can be obtained from $O(\lambda)=0[17,18]$, i.e.

$$
\begin{aligned}
L_{-2} \beta_{i j}= & E_{i j}^{\lambda}(\boldsymbol{x})-E_{i j}^{[0]}-E_{i k}^{\lambda}(\boldsymbol{x})\left(\partial \alpha_{j} / \partial y_{k}\right) \\
& -\partial\left(E_{k i}^{\lambda}(\boldsymbol{x}) \alpha_{j}\right) / \partial y_{k}
\end{aligned}
$$

Once functions $w^{[0]}, w^{[1]}$, and $w^{[2]}$ are obtained, they can be used to compute fine scale components of corresponding eigenvalues.

\section{Iterative corrector for 2 -scale eigensolution}

It will be shown in the numerical tests in Sect. 4 that 2-scale (scales [0] and [1]) asymptotic expansion yields considerable errors in the higher eigenmodes. Instead of introducing high order asymptotic expansion terms for desired accuracy, we consider the solution of the 2-scale expansion as the predictor of fine scale solution, and introduce power method and Rayleigh quotient iterative algorithm for correction of higher eigenmodes of the fine scale solution.

We begin with the discrete form of the homogenized eigenvalue equation in (2.27):

$\boldsymbol{K}^{[0]} \boldsymbol{u}^{[0]}=\omega^{[0]} \boldsymbol{M}^{[0]} \boldsymbol{u}^{[0]}$ in $\Omega$

where Dirichlet boundary conditions are imposed via the selection of admissible test and trial functions, $\boldsymbol{K}^{[0]}$ and $M^{[0]}$ are the matrices associated with the weak form operators $a(\cdot, \cdot)$ and $(\cdot, \cdot)$ of the homogenized problem defined in (2.10) and (2.11), and $\boldsymbol{u}^{[0]}$ is a coarse-scale eigenvector. The employed iterative scheme aims to find the eigenpairs $\left(\omega^{\lambda}, \boldsymbol{u}^{\lambda}\right)$ using the coarse-scale eigenpairs $\left(\omega^{[0]}, \boldsymbol{u}^{[0]}\right)$. The discrete form of the original multi-scale eigenvalue problem of Eq. (2.4) can be expressed as

$\boldsymbol{K}^{\lambda} \boldsymbol{u}^{\lambda}=\omega^{\lambda} \boldsymbol{M}^{\lambda} \boldsymbol{u}^{\lambda}$ in $\Omega$
Having the coarse scale component of each eigenpair, the initial guess (predictor) $\overline{\boldsymbol{u}}^{\lambda}(\boldsymbol{x}, \boldsymbol{y})$ as an approximation to $\boldsymbol{u}^{\lambda}(\boldsymbol{x}, \boldsymbol{y})$ is obtained from the 2 -scale asymptotic expansion

$\overline{\boldsymbol{u}}^{\lambda}(\boldsymbol{x}, \boldsymbol{y})=\boldsymbol{u}^{[0]}(\boldsymbol{x})+\lambda \boldsymbol{w}^{[1]}(\boldsymbol{x}, \boldsymbol{y})$

where $\boldsymbol{w}^{[1]}$ is obtained from Eq. (2.26). Correspondingly, initial guess for eigenvalues $\bar{\omega}^{\lambda}$ as an approximation to $\omega^{\lambda}$ is obtained by

$\bar{\omega}^{\lambda}=\omega^{[0]}+\lambda \bar{\omega}^{[1]}$

where $\bar{\omega}^{[1]}$ is the approximation of $\omega^{[1]}$ obtained from Eq. (2.19). Let the approximated eigenvector $\overline{\boldsymbol{u}}^{\lambda}(\boldsymbol{x}, \boldsymbol{y})$ be expressed by the $\boldsymbol{M}$-orthonomral eigenvectors $\left\{\boldsymbol{\psi}_{i}\right\}_{i=1}^{n}$ of the generalized eigenvalue problem (3.2) as

$\overline{\boldsymbol{u}}^{\lambda}=\sum_{i=1}^{n} \sigma_{i} \boldsymbol{\psi}_{i}$

in which $\sigma_{i}$ are the coefficients of the basis vectors. Using the power method [13] for eigenvalue solution, one premultiplies Eq. (3.5) by $\left(\boldsymbol{M}^{\lambda}\right)^{-m}\left(\boldsymbol{K}^{\lambda}\right)^{m}$, where $m$ is a positive integer, to yield

$$
\begin{aligned}
\left(\boldsymbol{M}^{\lambda}\right)^{-m}\left(\boldsymbol{K}^{\lambda}\right)^{m} \overline{\boldsymbol{u}}^{\lambda} & =\left(\boldsymbol{M}^{\lambda}\right)^{-m}\left(\boldsymbol{K}^{\lambda}\right)^{m}\left(\sum_{i=1}^{n} \sigma_{i} \boldsymbol{\psi}_{i}\right) \\
& =\sum_{i=1}^{n} \sigma_{i}\left(\omega_{i}^{\lambda}\right)^{m} \boldsymbol{\psi}_{i}
\end{aligned}
$$

Taking the norm of Eq. (3.6) we have

$$
\begin{aligned}
& \left\|\left(\boldsymbol{M}^{\lambda}\right)^{-m}\left(\boldsymbol{K}^{\lambda}\right)^{m} \overline{\boldsymbol{u}}^{\lambda}\right\| \\
& =\left|\sigma_{n}\right|\left(\omega_{n}^{\lambda}\right)^{m} \sqrt{1+\sum_{i=1}^{n-1}\left(\frac{\sigma_{i}}{\sigma_{n}}\right)^{2}\left(\frac{\omega_{i}^{\lambda}}{\omega_{n}^{\lambda}}\right)^{2 m}}
\end{aligned}
$$

where we assumed the eigenvalues are ordered $\omega_{n}^{\lambda}>\omega_{n-1}^{\lambda} \geq$ $\cdots \geq \omega_{2}^{\lambda}>\omega_{1}^{\lambda}$ and that the largest eigenvalue has multiplicity of one. Normalizing the resultant vector we have

$$
\begin{aligned}
& \left(\boldsymbol{M}^{\lambda}\right)^{-m}\left(\boldsymbol{K}^{\lambda}\right)^{m} \overline{\boldsymbol{u}}^{\lambda} /\left\|\left(\boldsymbol{M}^{\lambda}\right)^{-m}\left(\boldsymbol{K}^{\lambda}\right)^{m} \overline{\boldsymbol{u}}^{\lambda}\right\| \\
& =\frac{\sigma_{n}}{\left|\sigma_{n}\right|}\left[\sqrt{1+\sum_{i=1}^{n-1}\left(\frac{\sigma_{i}}{\sigma_{n}}\right)^{2}\left(\frac{\omega_{i}^{\lambda}}{\omega_{n}^{\lambda}}\right)^{2 m}}\right]^{-1} \boldsymbol{\psi}_{n} \\
& \quad+\sum_{i=1}^{n-1} \frac{\sigma_{i}}{\left|\sigma_{n}\right|}\left(\frac{\omega_{i}^{\lambda}}{\omega_{n}^{\lambda}}\right)^{m}\left[\sqrt{1+\sum_{j=1}^{n-1}\left(\frac{\sigma_{j}}{\sigma_{n}}\right)^{2}\left(\frac{\omega_{j}^{\lambda}}{\omega_{n}^{\lambda}}\right)^{2 m}}\right]^{-1} \boldsymbol{\psi}_{i}
\end{aligned}
$$

It can be shown that in this power method as $m \rightarrow \infty$,

$$
\lim _{m \rightarrow \infty}\left(\boldsymbol{M}^{\lambda}\right)^{-m}\left(\boldsymbol{K}^{\lambda}\right)^{m} \overline{\boldsymbol{u}}^{\lambda} /\left\|\left(\boldsymbol{M}^{\lambda}\right)^{-m}\left(\boldsymbol{K}^{\lambda}\right)^{m} \overline{\boldsymbol{u}}^{\lambda}\right\|=\boldsymbol{\psi}_{n}
$$


where $\psi_{n}$ is the eigenvector associated with the largest eigenvalue. Other eigenvectors can be obtained by GramSchmidt orthogonalization.

In practical problems, it is more desirable to obtain the first few smallest eigenvalues and the corresponding eigenvectors. An alternative form of power method (inverse iteration) is to replace $\left(\boldsymbol{M}^{\lambda}\right)^{-1}\left(\boldsymbol{K}^{\lambda}\right)$ by $\left(\boldsymbol{K}^{\lambda}-\bar{\omega}_{1}^{\lambda} \boldsymbol{M}^{\lambda}\right)^{-1}$, where $\bar{\omega}_{1}^{\lambda}$ is the approximation of the smallest eigenvalue. It can be shown

$$
\begin{aligned}
& \left(\boldsymbol{K}^{\lambda}-\bar{\omega}_{1}^{\lambda} \boldsymbol{M}^{\lambda}\right)^{-m} \overline{\boldsymbol{u}}_{\lambda} /\left\|\left(\boldsymbol{K}^{\lambda}-\bar{\omega}_{1}^{\lambda} \boldsymbol{M}^{\lambda}\right)^{-m} \overline{\boldsymbol{u}}_{\lambda}\right\| \\
& =\frac{\sigma_{1}}{\left|\sigma_{1}\right|}\left[\sqrt{1+\sum_{j=2}^{n}\left(\frac{\sigma_{j}}{\sigma_{1}}\right)^{2}\left(\frac{\omega_{j}^{\lambda}-\bar{\omega}_{1}^{\lambda}}{\omega_{1}^{\lambda}-\bar{\omega}_{1}^{\lambda}}\right)^{-2 m}}\right]^{-1} \boldsymbol{\psi}_{1} \\
& +\sum_{i=2}^{n} \frac{\sigma_{i}}{\left|\sigma_{1}\right|}\left(\frac{\omega_{i}^{\lambda}-\bar{\omega}_{1}^{\lambda}}{\omega_{1}^{\lambda}-\bar{\omega}_{1}^{\lambda}}\right)^{-m} \\
& \quad \times\left[\sqrt{1+\sum_{j=2}^{n}\left(\frac{\sigma_{j}}{\sigma_{1}}\right)^{2}\left(\frac{\omega_{j}^{\lambda}-\bar{\omega}_{1}^{\lambda}}{\omega_{1}^{\lambda}-\bar{\omega}_{1}^{\lambda}}\right)^{-2 m}}\right]^{-1} \boldsymbol{\psi}_{i}
\end{aligned}
$$

It can be seen that if $\omega_{1}^{\lambda}-\bar{\omega}_{1}^{\lambda} \approx 0$ and $\sigma_{1}$ is not too small, we have

$\lim _{m \rightarrow \infty}\left(\boldsymbol{K}^{\lambda}-\bar{\omega}_{1}^{\lambda} \boldsymbol{M}^{\lambda}\right)^{-m} \overline{\boldsymbol{u}}^{\lambda} /\left\|\left(\boldsymbol{K}^{\lambda}-\bar{\omega}_{1}^{\lambda} \boldsymbol{M}^{\lambda}\right)^{-m} \overline{\boldsymbol{u}}^{\lambda}\right\|=\boldsymbol{\psi}_{1}$

Similarly, other eigenvectors can be obtained by GramSchmidt orthogonalization.
For larger system of equations, one can combine the above methods with Rayleigh quotient iteration which has cubic rate of convergence. Assuming $\boldsymbol{K}^{\lambda}, \boldsymbol{M}^{\lambda}$ are symmetric, and initial guess $\overline{\boldsymbol{u}}^{\lambda}$ is a given non-zero vector, it can be shown that the Rayleigh quotient minimizes $\left\|\left(\boldsymbol{K}^{\lambda}-\omega \boldsymbol{M}^{\lambda}\right) \overline{\boldsymbol{u}}^{\lambda}\right\|$ where the Rayleigh quotient is defined by:

$\omega=\frac{\left(\overline{\boldsymbol{u}}^{\lambda}\right)^{\mathrm{T}} \boldsymbol{K}^{\lambda} \overline{\boldsymbol{u}}^{\lambda}}{\left(\overline{\boldsymbol{u}}^{\lambda}\right)^{\mathrm{T}} \boldsymbol{M}^{\lambda} \overline{\boldsymbol{u}}^{\lambda}}$

The inverse iteration and Rayleigh quotient are combined to correct the 2-scale asymptotic expansion solution. Using the 2-scale asymptotic expansion solution as the initial guess of the eigensolution, inverse iterations are first employed to obtain the ordered approximate eigenvectors. Note that at this stage the inverse iteration is mainly used to sort out the order of the approximated eigensolutions and thus the order $m$ in Eq. (3.10) does not have to be very high. Our numerical test indicates that $m=5$ is sufficient. Thereafter, Rayleigh quotient is used to correct the eigenvalues and inverse iteration is further employed to correct the eigenvectors. The computational procedures of the proposed iterative scheme as a correction of the 2-scale asymptotic expansion solution are given in Table 1.

\section{Numerical results}

Two examples are analyzed using finite element method to solve eigenvalue problems with rapidly oscillating periodic and random coefficients using the proposed method. The

Table 1 Iterative asymptotic expansion eigenvalue solution procedures

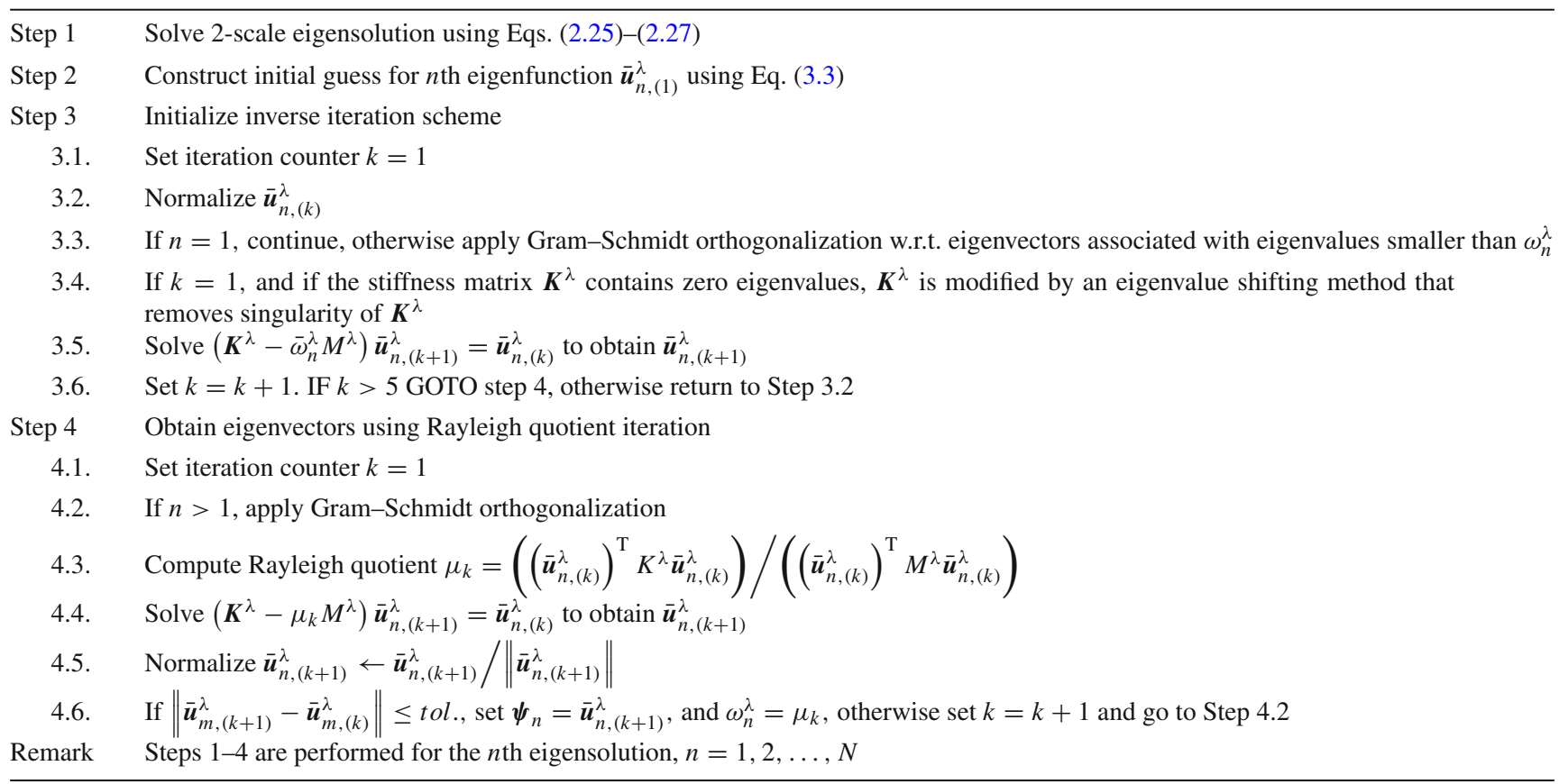


reference solutions $u^{\lambda}$ for comparison with the 2-scale solution are obtained from the single-scale calculation using very fine mesh. In this approach, $u^{[0]}+u^{[1]}$ is the approximation of $u^{\lambda}$ based on 2-scale asymptotic expansion as the predictor, and the combined inverse iteration and Rayleigh quotient iteration are used as the correction of the predictor. Thus the error of this approach is resulting from the first order approximation in the 2-scale asymptotic expansion (since higher order fine scale solution is not considered) and the iteration correction of this first order expansion.

\subsection{Elliptic eigenvalue problem with periodic coefficients}

An eigenvalue problem is given by

$\left.-\frac{\partial}{\partial x}\left(E^{\lambda}(x) \frac{\partial u^{\lambda}(x)}{\partial x}\right)=\omega^{\lambda} u^{\lambda}(x), \quad x \in\right] 0,1[$

$u_{, x}^{\lambda}(0)=u_{, x}^{\lambda}(1)=0$

where $E^{\lambda}(x)$ are rapidly oscillating periodic coefficients as shown in Fig. 1. The whole domain consists of 5 unit cells, each of which consists of 40 intervals with oscillating coefficients. The 2-scale auxiliary problem is solved first. Thereafter, scales [0] and [1] components of auxiliary function $w^{\lambda}$ are obtained. Once the 2-scale components of the auxiliary function are obtained, predictor equation is employed to obtain the initial guess of the eigenvalues and eigenfunctions. For the 2-scale approach, 40 quadratic elements are used in the unit cell, and 20 quadratic elements are used for scale [0] solution. A single-scale model with 200 quadratic elements is constructed for obtaining a reference solution. Note that in the 2-scale approach the eigenvalue problem is only solved at scale [0] (20 elements), while in the single-scale approach the eigenvalue problem needs to be solved with much more degrees of freedom.

The iterative procedures are employed to correct the 2-scale asymptotic expansion solution. Relative errors of

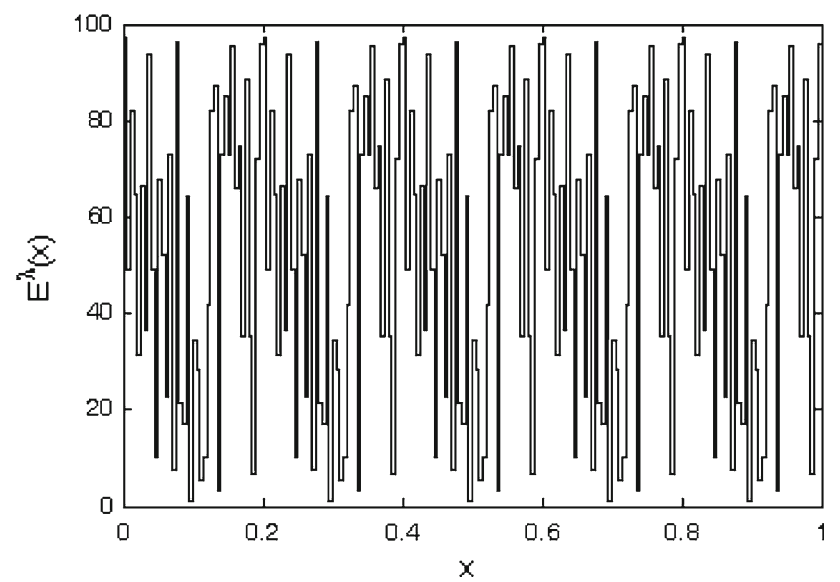

Fig. 1 Periodic rapidly oscillating coefficients

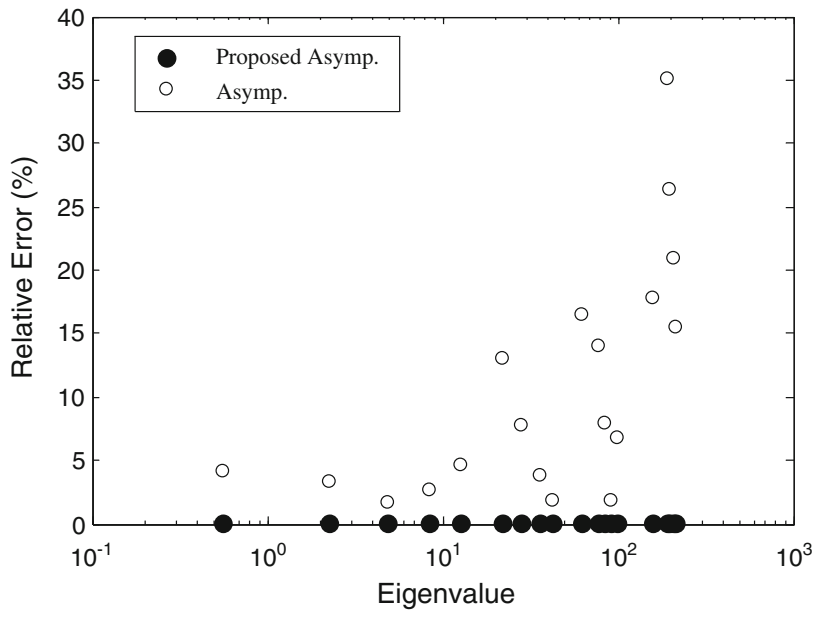

Fig. 2 Relative error in eigenvalues obtained from the 2-scale asymptotic and the proposed corrected 2 -scale asymptotic methods

the first 20 eigenvalues and eigenfunctions calculated by the 2 -scale asymptotic expansion method and the proposed asymptotic expansion with iteration correction are shown in Fig. 2. The results show that the proposed iterative scheme significantly reduces the errors in the eigenvalues obtained by the 2-scale asymptotic expansion method. Moreover, Fig. 3 demonstrates that by comparison with [13] where only coarse-scale component of the eigenfunction was utilized as the predictor and corrected by the power method, the number of iterations is substantially less in the proposed method. The eigenfunctions obtained from 2-scale asymptotic expansion are shown in Fig. 4, where large errors exist in the high modes when compared with the reference solution obtained from single-scale calculation with very fine mesh. These errors can be significantly reduced when the proposed iterative procedures are used as shown in Fig. 5.

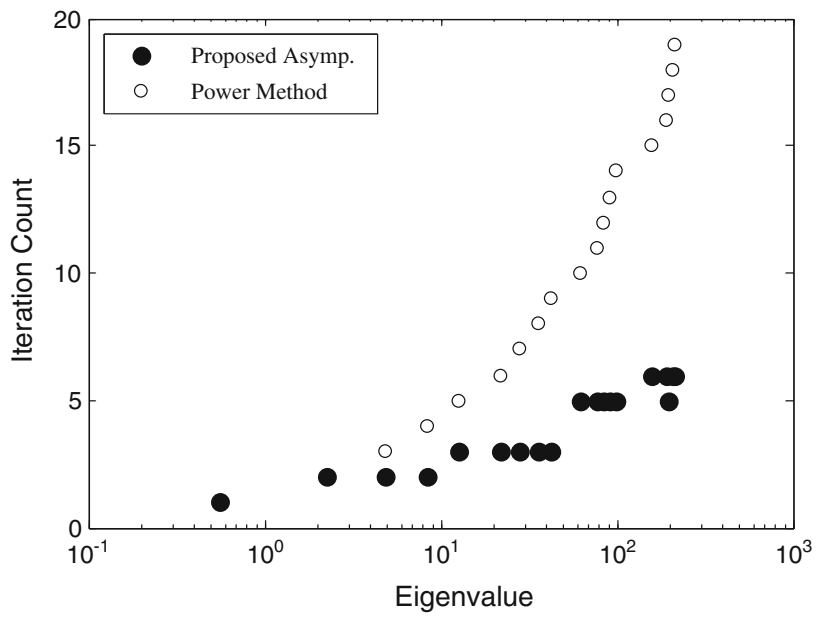

Fig. 3 Comparison of iteration counts in the power method and the proposed method enhanced by the correction of coarse-scale solution 

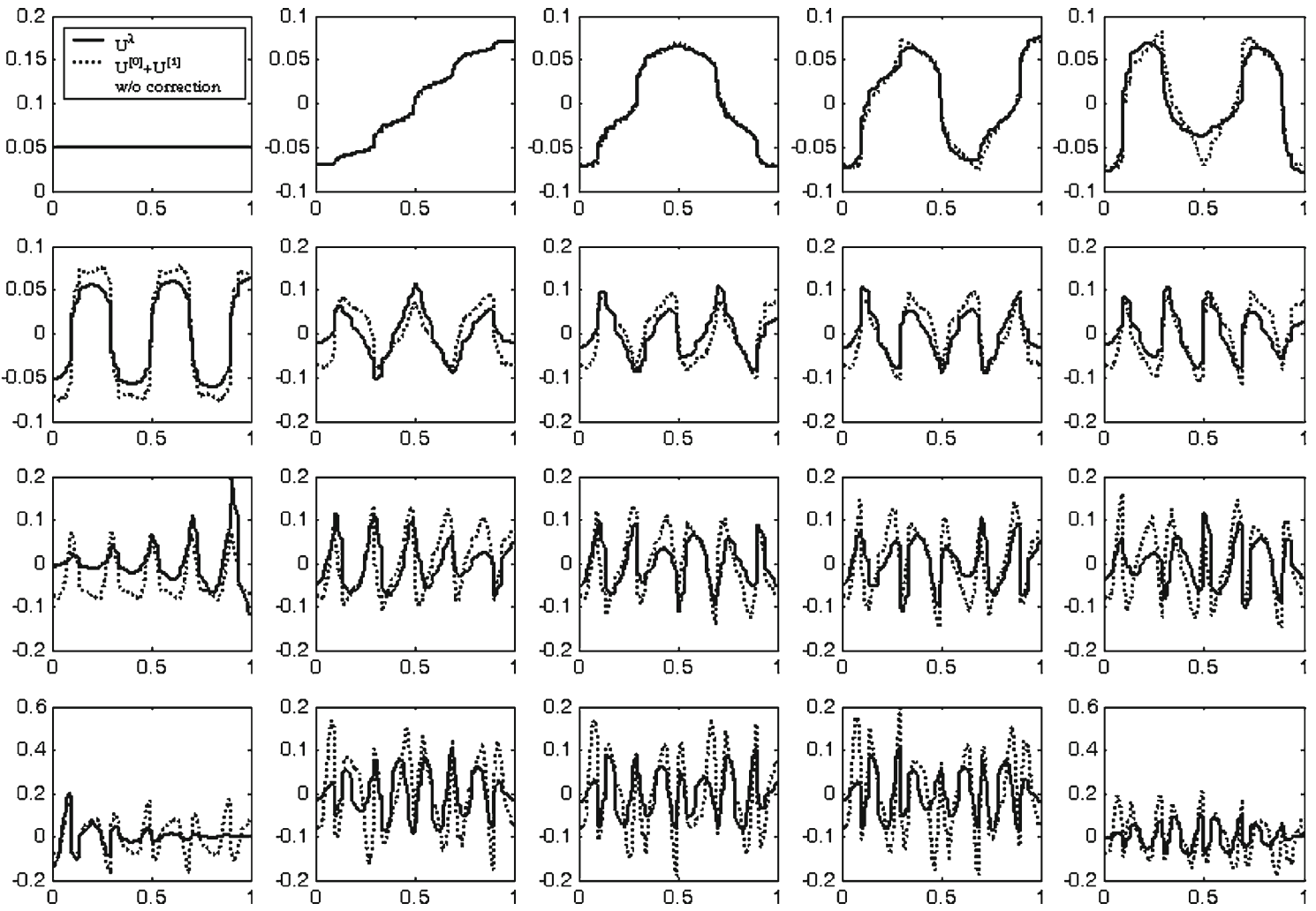

Fig. 4 Comparison of the first 20 eigenfunctions obtained from 2-scale asymptotic expansion method and the reference solution
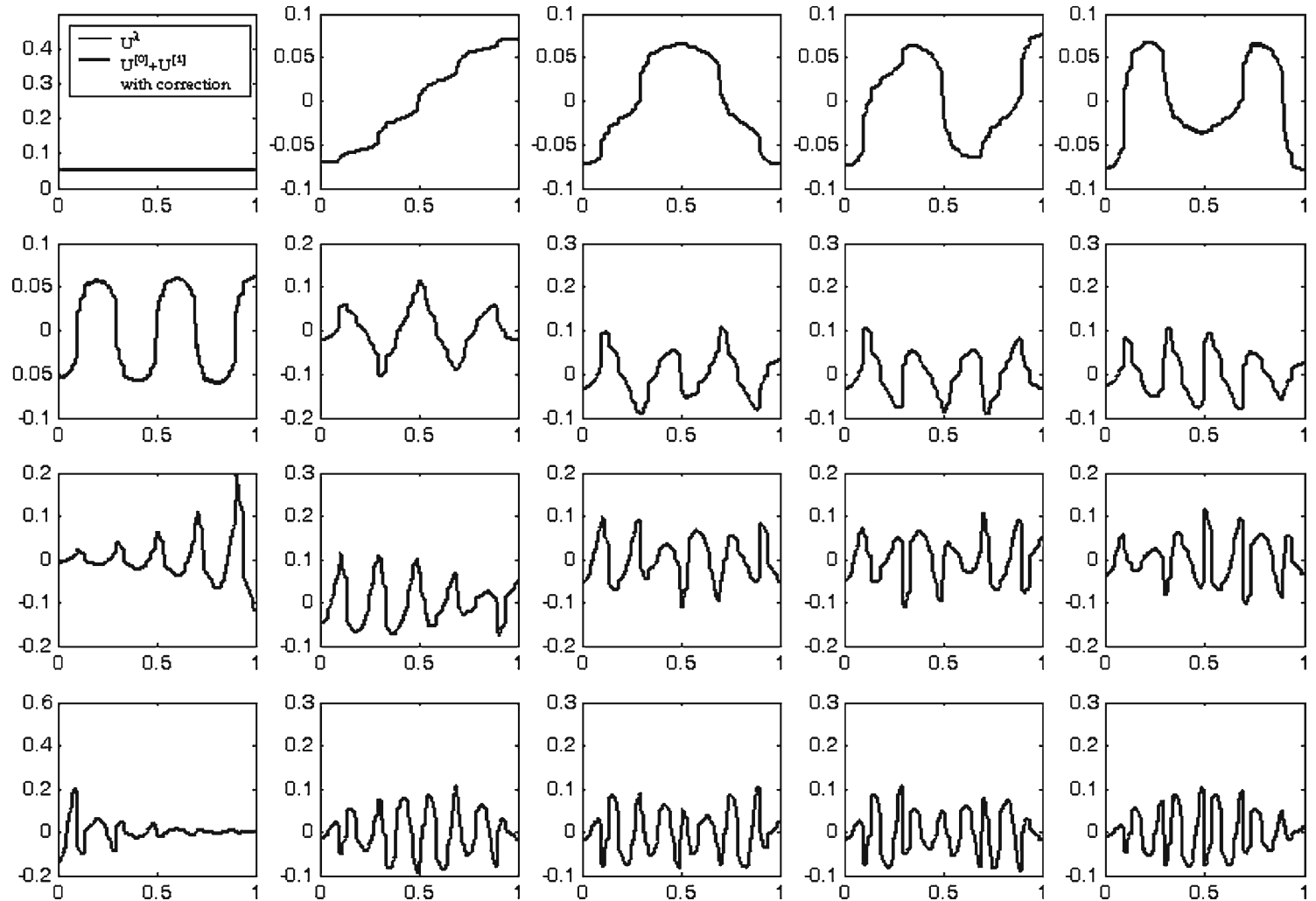

Fig. 5 Comparison of the first 20 eigenfunctions obtained from iterative 2-scale asymptotic expansion method and the reference solution 
It is also observed that the condition numbers of the matrices in the scale [0] equation are much smaller than the condition numbers of the matrices in the fine single-scale model when the heterogeneity of the eigenvalue problem is significant (when the oscillation in $E_{i j}^{\lambda}$ is sufficiently high). The condition number is defined as $\lambda_{\max } / \lambda_{\min }$, where $\lambda_{\max }$ and $\lambda_{\min }$ are the maximum and minimum eigenvalues of a matrix, respectively. It is noted that the error of the solution of a system of algebraic equations is linearly proportional to the condition number of the matrix associated with the algebraic equations [7]. The matrix with very large condition number is referred to as an ill-conditioned matrix. In this example, the single-scale approach for problems with highly oscillating coefficients yields a relatively ill-conditioned discrete system (with much larger condition numbers) compared to the 2-scale approach. The condition numbers of the matrices in the eigenvalue problems are $3.121 \mathrm{e}+5$ and $9.069 \mathrm{e}+2$ for single-scale and 2-scale approaches, respectively.

\subsection{Elliptic eigenvalue problem with random coefficients}

In this example, eigenvalue problem in Eq. (4.1) with random heterogeneity as shown in Fig. 6 is analyzed. We treat the whole problem domain as one unit cell, and assume that this unit cell is periodically arranged in space. For the 2 -scale approach, 200 quadratic elements are used in the unit cell, and 20 quadratic elements are used for the scale [0] solution. A single-scale model with 200 quadratic elements is constructed for obtaining a reference solution. Note that in the 2-scale approach the eigenvalue problem is solved at the scale [0] only (with 20 elements), while in the singlescale approach the eigenvalue problem needs to be solved with much more degrees of freedom. As shown in Fig. 7, for this problem with random heterogeneity, errors in the eigensolution obtained from the 2-scale asymptotic expansion method can also be significantly reduced by the proposed

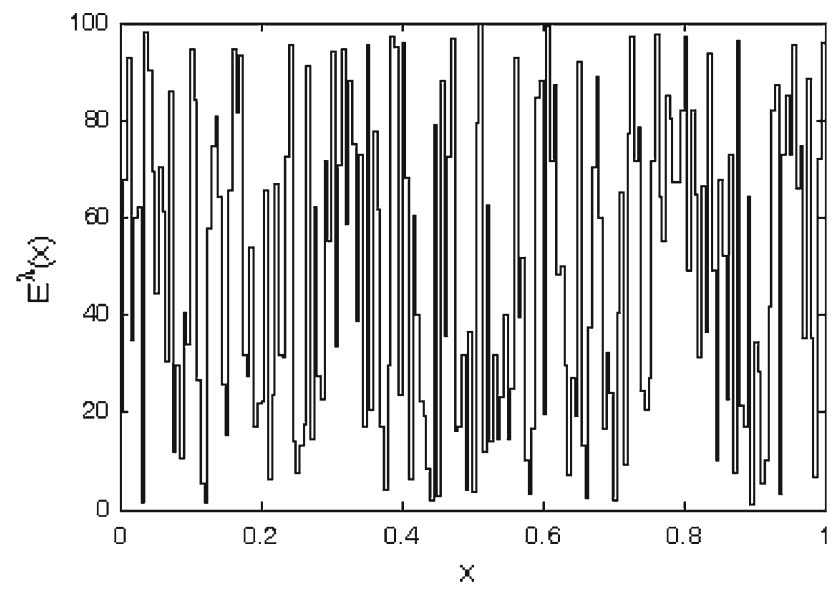

Fig. 6 Non-periodic rapidly oscillating coefficients

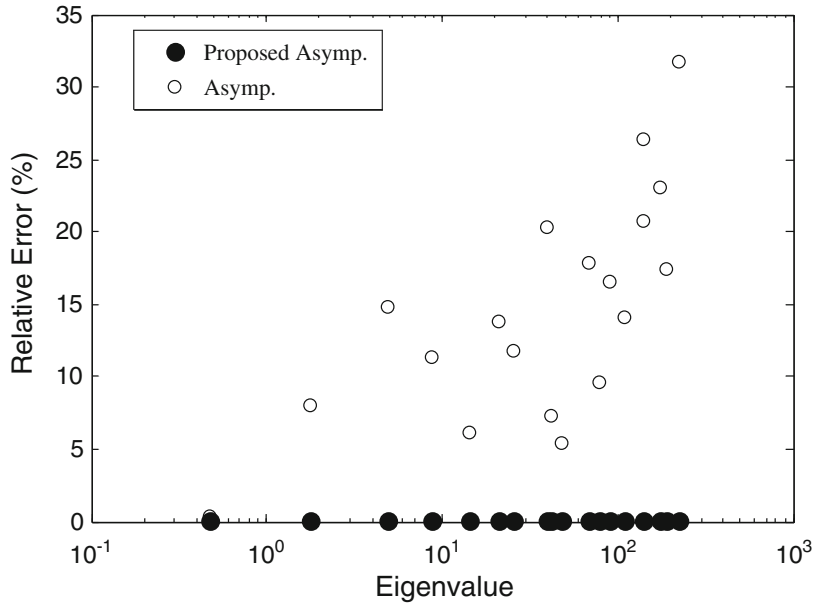

Fig. 7 Relative error in eigenvalues obtained from the 2-scale asymptotic and the proposed corrected 2 -scale asymptotic methods

iterative 2-scale asymptotic expansion method. The number of iterations required in the proposed method is considerably less than that required using the power method as illustrated in Fig. 8. Furthermore, as shown in Figs. 9 and 10, errors in the eigenfunctions obtained from the 2-scale asymptotic expansion method are dramatically reduced by the proposed iterative 2-scale asymptotic expansion method, particularly in the higher modes.

As mentioned in Example 4.1, the single-scale approach for problems with highly oscillating coefficients yields a relatively ill-conditioned discrete system (with much larger condition numbers) compared to the 2 -scale approach. In this example, the condition numbers of the matrices in the eigenvalue problems are $4.098 \mathrm{e}+5$ and $9.069 \mathrm{e}+2$ for single-scale and 2-scale approaches, respectively.

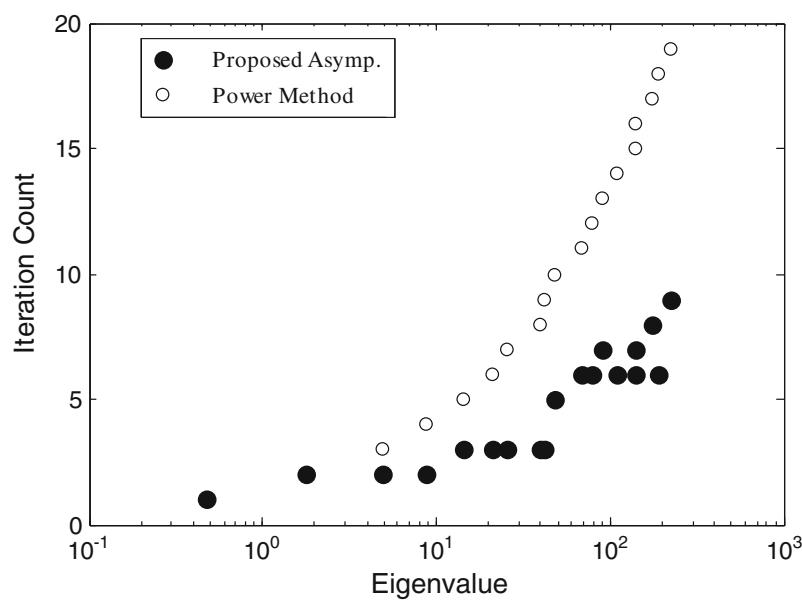

Fig. 8 Iteration counts in iterative solver and power method for 2-scale eigensolution 

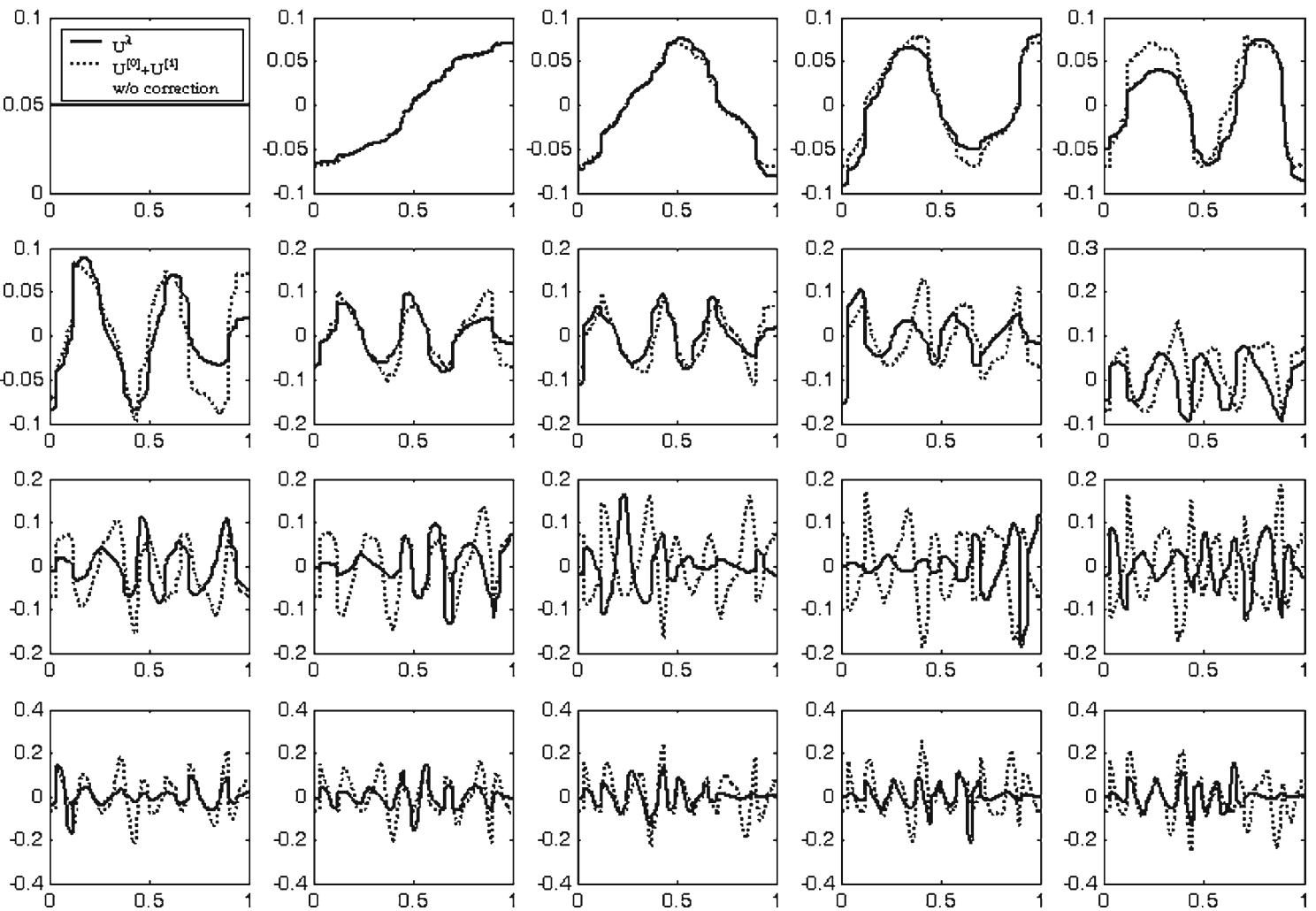

Fig. 9 Comparison of the first 20 eigenfunctions obtained from 2-scale asymptotic expansion method and reference solution
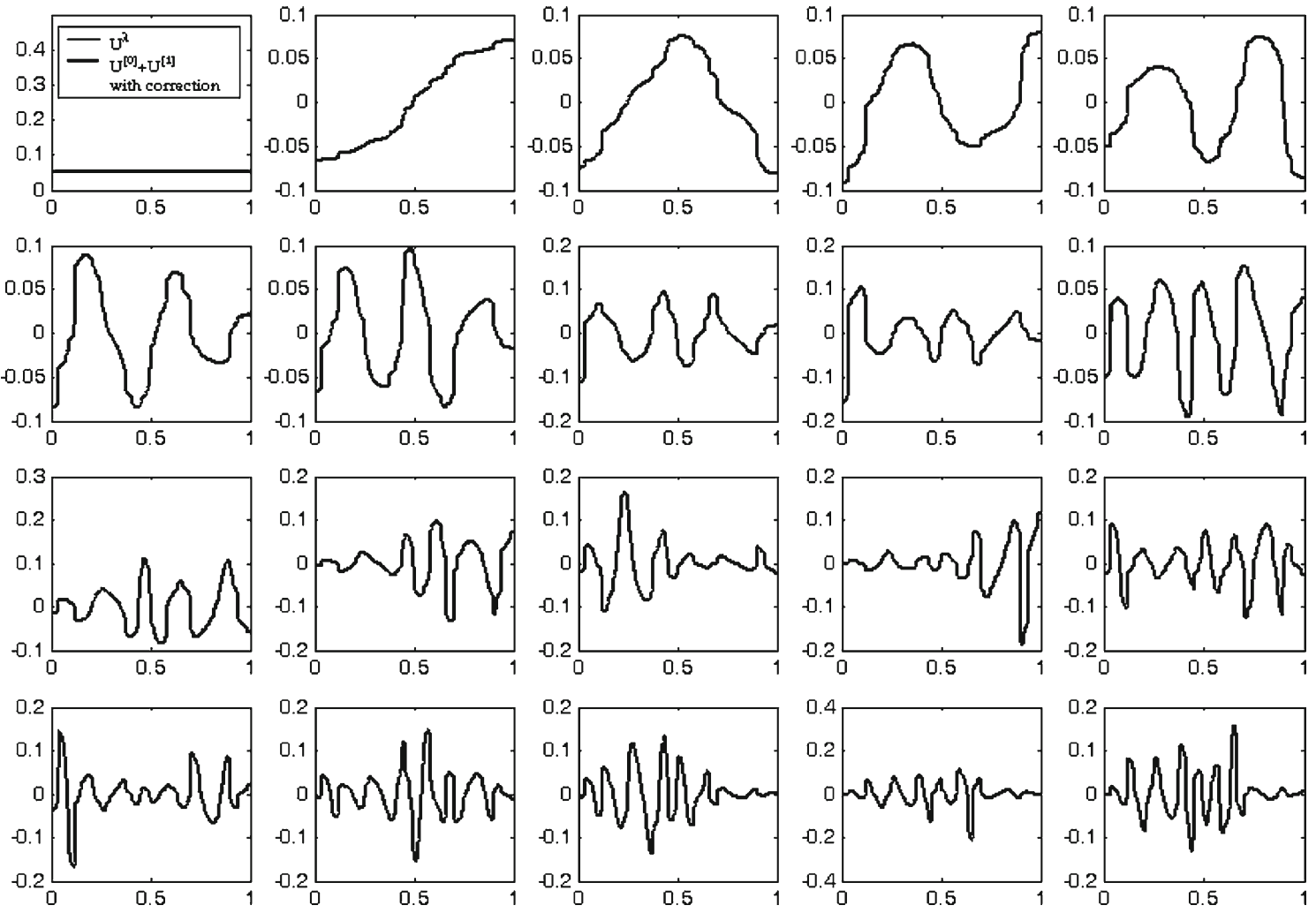

Fig. 10 Comparison of the first 20 eigenfunctions obtained from the iterative asymptotic expansion method and the reference solution 
Fig. 11 2D composite material model. a Full model, b unit model, c discretization

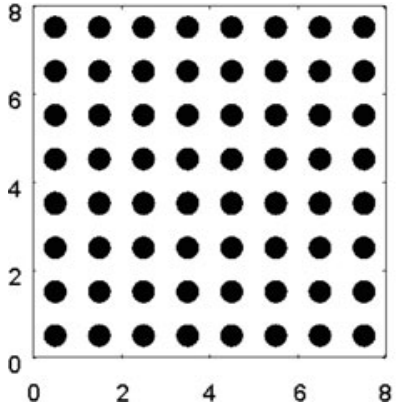

(a)

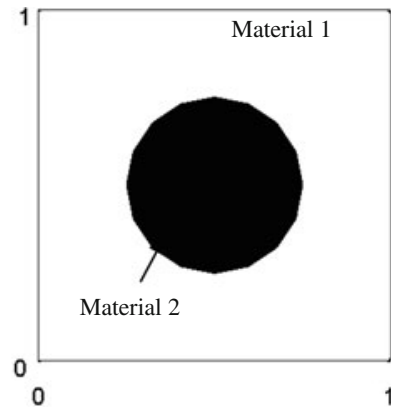

(b)

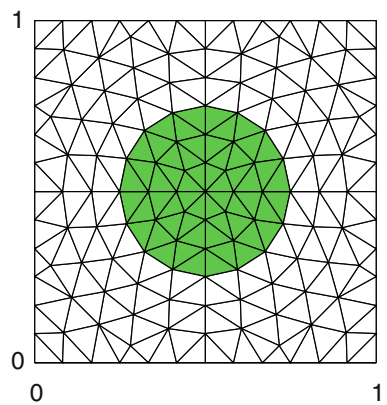

(c)

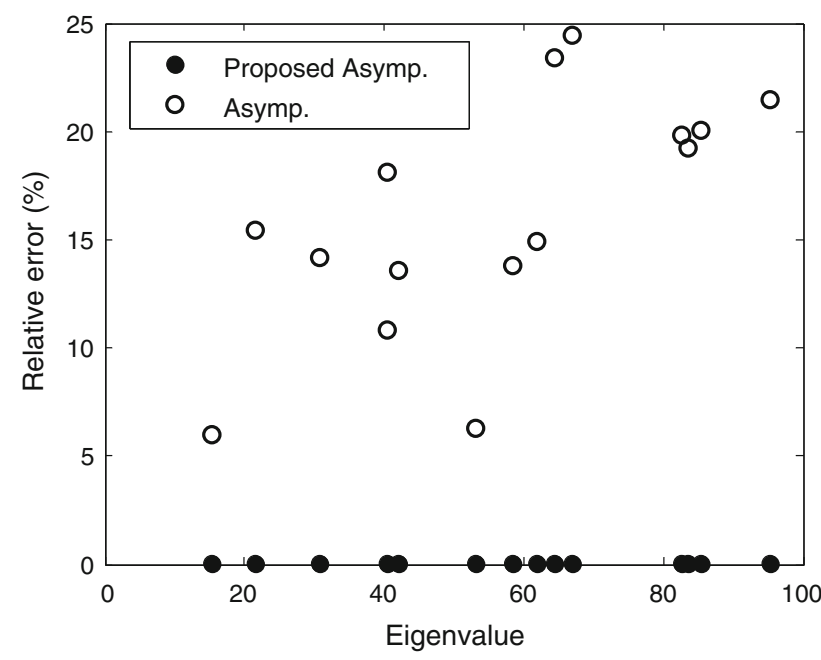

Fig. 12 Relative error in eigenvalues obtained from iterative 2-scale asymptotic expansion method

\subsection{Eigenvalue analysis of a 2-dimensional composite}

A 2-dimensional Dirichlet eigenvalue problem is given by

$$
\begin{aligned}
& -\frac{\partial}{\partial x_{j}}\left(C_{i j k l}^{\lambda} \frac{\partial u_{k}^{\lambda}}{\partial x_{l}}\right)=\omega^{\lambda} u_{i}^{\lambda},(i, j, k, l=1,2) \text { in } \Omega \\
& u_{i}^{\lambda}=0,(i=1,2) \text { on } \Gamma
\end{aligned}
$$

where $C_{i j k l}^{\lambda}$ is material tensor, which is periodic and oscillating due to material inhomogeneity. The composite under consideration is shown in Fig. 11. The Young's modulus and Poisson ratio of two materials are $E_{1}=10,000, v_{1}=$ $0.3, E_{2}=1,000, v_{2}=0.1$. The discretization of the unit cell is shown in Fig. 11c. For the solution of scale [0], 16 $\times 16$ bi-linear elements are used, and linear triangular elements with mesh shown in Fig. 11c are used in the unit cell. For validation of the 2-scale solution strategy, a single-scale model using the same discretization of the unit cell for the whole domain is generated. The eigensolution of this finescale model is used as the reference solution.

We first compare the solution accuracy of the methods based on 2-scale asymptotic expansion and the proposed

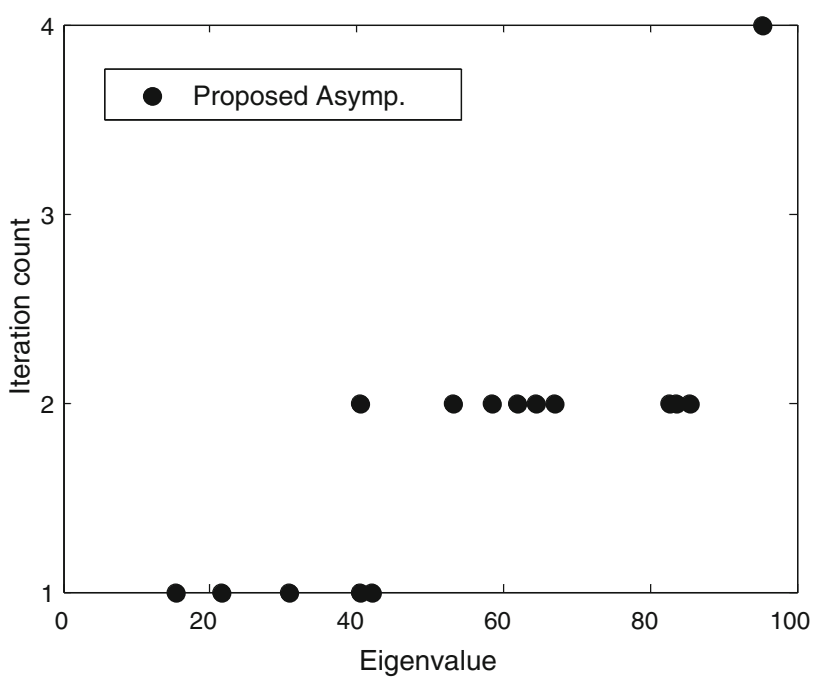

Fig. 13 Iteration counts in iterative 2-scale eigensolution

iterative 2-scale asymptotic expansion. As shown in Fig. 12, the proposed method greatly reduces the error in the eigenvalues obtained from the 2 -scale asymptotic expansion method. The number of iterations required in the proposed method is plotted in Fig. 13, where the convergence tolerance is set to be $10^{-5}$. Figure 14 shows the selected eigenfunctions obtained by the proposed iterative 2 -scale method. The comparison of the eigenmodes obtained by the iterative 2-scale method and the single-scale model along the diagonal line of the domain given in Fig. 15 shows satisfactory agreement.

\section{Conclusion}

Solving an eigenvalue problem with highly oscillatory coefficient in the differential operator is computationally challenging with single-scale calculation. In this work, an iterative predictor-corrector approach was introduced to allow construction of fine-scale eigenvalues and eigenfunctions using the coarse-scale solution.

We first introduced an asymptotic expansion of the eigenvalue problem. By employing an auxiliary weak formulation of the original eigenvalue problem, a predictor 


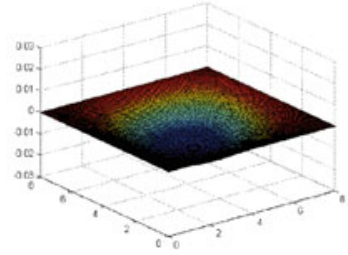

$u_{x}^{[0]}$

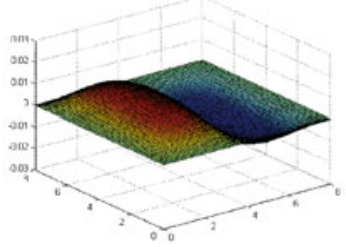

$u_{x}^{[0]}$

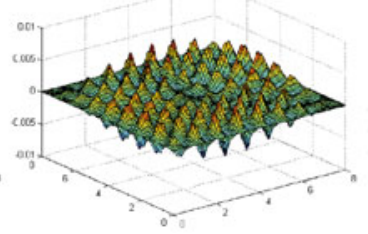

$u_{x}^{[1]}$

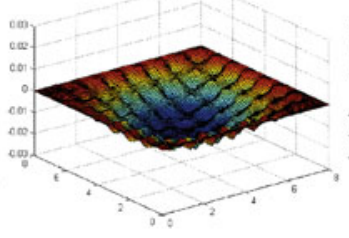

$u_{x}^{[0]}+u_{x}^{[1]}$

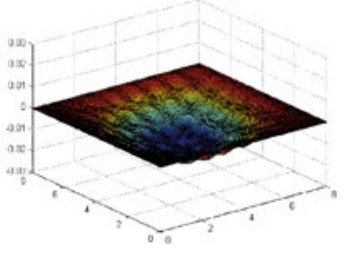

$u_{x}^{[0]}+{ }_{u_{x}}^{[1]} \backslash \mathrm{W}$ correction

(a)

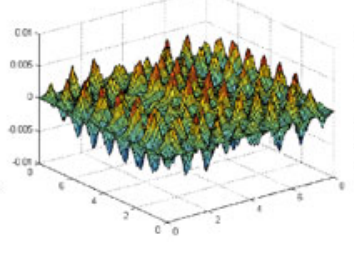

$u_{x}^{[1]}$

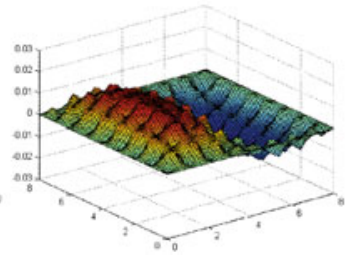

$u_{x}^{[0]}+u_{x}^{[1]}$

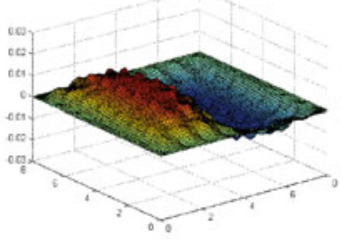

${ }_{u_{x}}^{[0]}+{ }_{u_{x}}^{[1]} \backslash \mathrm{W}$ correction

(b)
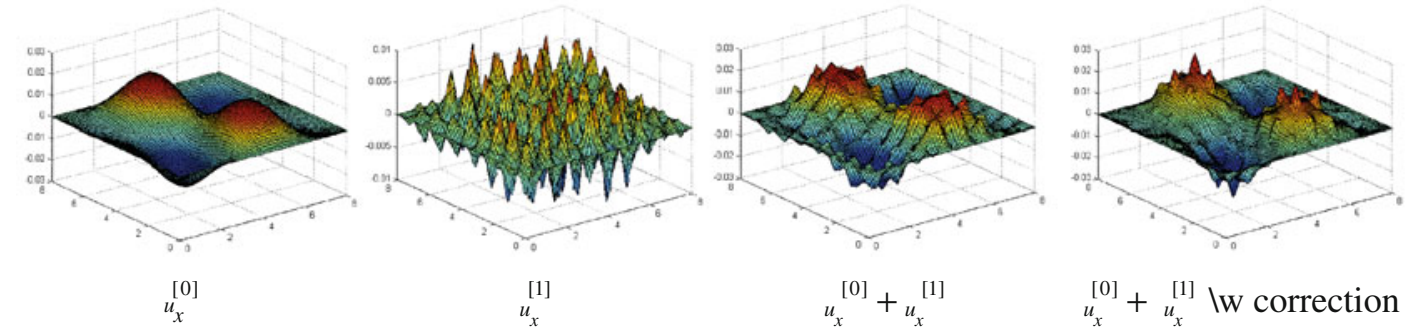

$u_{x}^{[0]}+{ }_{u_{x}}^{[1]}$

${ }_{u_{x}}^{[0]}+{ }_{u_{x}}^{[1]} \mid \mathrm{W}$ correction

(c)
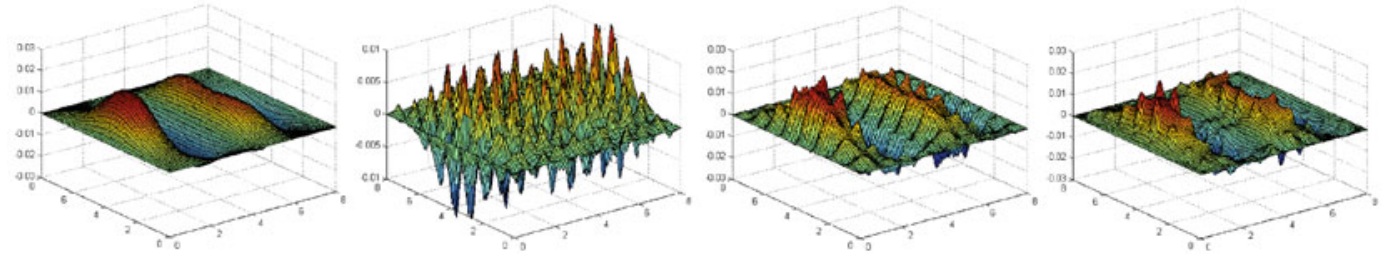

$u_{x}^{[0]}$

$u_{x}^{[1]}$

$u_{x}^{[0]}+u_{x}^{[1]}$

${ }_{u_{x}}^{[0]}+{ }_{u_{x}}^{[1]} \mid \mathrm{W}$ correction

(d)

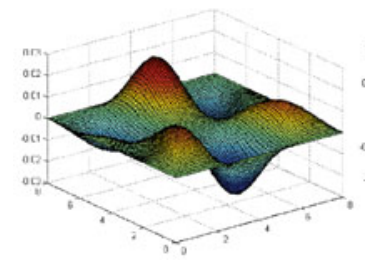

$[0]$
$u_{x}$

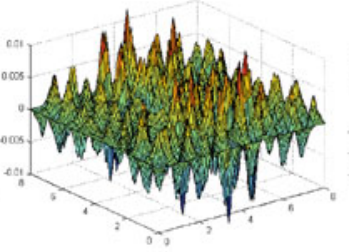

$u_{x}^{[1]}$

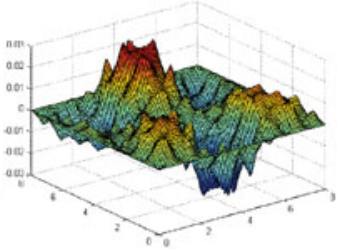

$u_{x}^{[0]}+{ }_{u_{x}}^{[1]}$

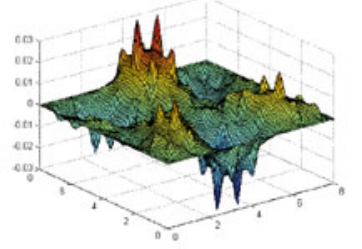

$u_{x}^{[0]}+u_{x}^{[1]} \mid$ W correction

(e)

Fig. 14 Selected eigenfunctions of displacement $u_{x}$ obtained from the iterative asymptotic expansion method: a 1st eigenfunction, $\mathbf{b}$ 4th eigenfunction, $\mathbf{c} 10$ th eigenfunction, $\mathbf{d} 13$ th eigenfunction, e 16th eigenfunction 

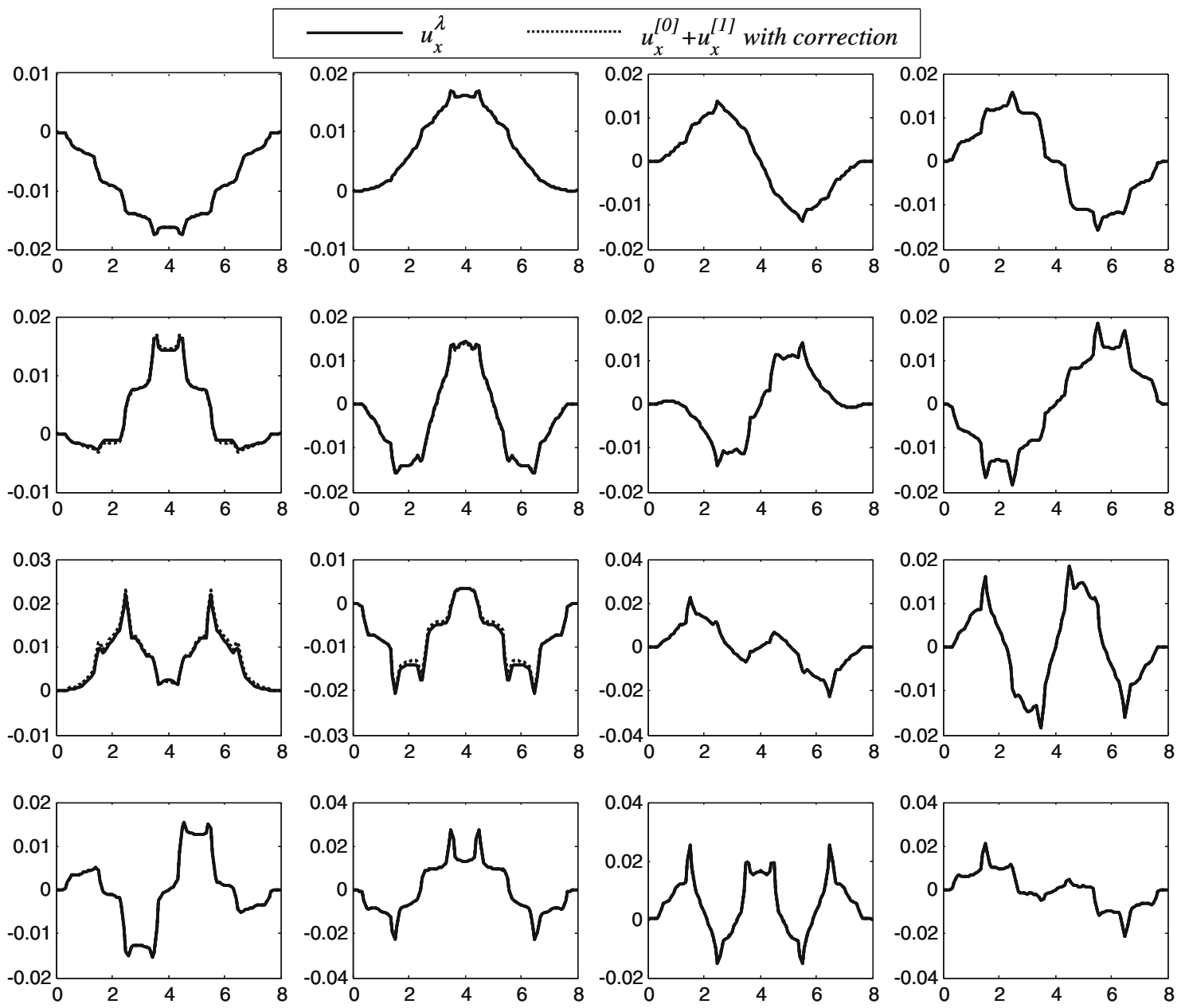

Fig. 15 Comparison of the first 16 eigenfunctions of displacement $u_{x}$ obtained from the iterative asymptotic expansion method and the reference solution along diagonal line of the problem domain

equation was obtained. This predictor equation offers a relationship between the multi-scale eigenvalues and eigenfunctions through an auxiliary function. By introducing an asymptotic expansion of the auxiliary function and the differential operator, a set of leading order equations were obtained for solving coarse-scale and fine-scale auxiliary functions. Using the auxiliary functions in conjunction with the predictor equation, the multi-scale eigenvalues and eigenvectors can be computed.

It was shown, however, that the asymptotic expansion of the auxiliary eigenvalue problem with 2 scales was insufficient in obtaining accurate higher eigenmodes. We considered the 2-scale asymptotic expansion solution as an initial guess of the eigensolution, and introduced an iterative scheme as a correction of the predictor solution. This iterative scheme was composed of an inverse iteration and a Rayleigh quotient iteration. The inverse iteration was utilized to keep the lowest to highest eigensolutions in order, while the
Rayleigh quotient iteration was employed to accelerate the rate of convergence of the iteration.

Elliptic eigenvalue problem with periodic and random coefficients were analyzed using the proposed method. It was illustrated that although the asymptotic expansion of the auxiliary problem allows obtaining the fine-scale eigensolution based on the coarse-scale eigensolution, large errors exist in the higher eigenmodes when the 2-scale asymptotic expansion was used. These errors can be effectively reduced by correcting the 2-scale asymptotic expansion solution using the proposed inverse iteration and Rayleigh quotient iteration. It was also shown that the proposed iterative scheme requires considerably less iterations compared to the power method.

Open Access This article is distributed under the terms of the Creative Commons Attribution Noncommercial License which permits any noncommercial use, distribution, and reproduction in any medium, provided the original author(s) and source are credited. 


\section{References}

1. Albert F, Charru R (2000) Small Reynolds number instabilities in two-layer Couette flow. Eur J Mech B Fluids 19:229-252

2. Bathe K-J (1996) Finite element procedures. Prentice-Hall, Englewood Cliffs

3. Bensoussan A, Lions JL, Papanicolau G (1978) Asymptotic analysis for periodic sturctures. North Holland, Amsterdam

4. Cao LQ, Cui JZ, Zhu DC (2002) Multiscale asymptotic analysis and numerical simulation for the second order Helmholtz equation with oscillating coefficients over general convex domains. SIAM J Numer Anal 40:543-577

5. Cao LQ, Cui JZ (2004) Asymptotic expansions and numerical algorithms of eigenvalues and eigenfunctions of the Dirichlet problem for second order elliptic equations in perforated domains. Numer Math 96:525-581

6. de Hoog FR, Anderssen RS (2001) Asymptotic formulas for discrete eigenvalue problems in Liouville normal form. Math Models Methods Appl Sci 11(1):43-56

7. Demmel JW (1997) Applied numerical linear algebra. SIAM, Philadephia

8. Dyka CT, Ingel RP, Flippen LD (1996) A new approach to dynamic condensation for FEM. Comput Sturct 61(4):763-773

9. Flippen LD (1992) Polynomial-basis model reduction. Math Comput Model 16(12):121-132

10. Flippen LD (1995) Interpolation-based condensation of algebraic semi-discrete models with frequency response application. Comput Math Appl 29(9):39-52

11. Flippen LD (1994) A theory of condensation model reduction. Comput Math Appl 27(2):9-40

12. Gregory B, Nicholas C (1998) A multiresolution strategy for reduction of elliptic PDEs and eigenvalue problems. Appl Comput Harmon Anal 5:129-155

13. Golub GH, Van Loan CF (1996) Matrix computations. The Johns Hopkins University Press, Baltimore

14. Igusa T, Achenbach JD, Min KW (1991) Resonance characteristics of connected subsystems: theory and simple configurations. J Sound Vib 146(3):407-421

15. Ingel RP, Dyka CT, Flippen LD (2000) Model reduction and frequency windowing for acoustic FEM analysis. J Sound Vib 238(2):327-350
16. Kamiński M (2005) Homogenization-based finite element analysis of unidirectional composites by classical and multiresolutional techniques. Comput Methods Appl Mech Eng 194:2147-2173

17. Kesavan S (1979) Homogenization of elliptic eigenvalue problems, Part 1. Appl Math Optim 5:153-167

18. Kesavan S (1979) Homogenization of elliptic eigenvalue problems, Part 2. Appl Math Optim 5:197-216

19. Knapek S (1999) Matrix-dependent multigrid-homogenization for diffusion problems, unpublished manuscript

20. Leung AY (1978) An accurate method of dynamic condensation in structural analysis. Int J Numer Methods Eng 12:1705-1715

21. Mandel J, McCormick S (1989) A multilevel variational method for $A u=\lambda B u$ on composite grids. J Comput Phys 80:442-452

22. Moskow S, Vogelius M (1997) First-order corrections to the homogenized eigenvalues of a periodic composite medium, a convergence proof. Proc R Soc Edinb 127A:1263-1299

23. Rajendran S, Raghavan MR, Narasimhan MV (1995) Improving the convergence rate of inverse iteration method. Comput Struct 55:709-715

24. Rajendran S, Narasimhan MV (1994) An accelerated subspace iteration method. Int J Numer Methods Eng 37:141-153

25. Rajendran S (2002) Computing the lowest eigenvalue with Rayleigh quotient iteration. J Sound Vib 254(3):599-612

26. Randles PW (1992) Synthesis of equivalent dynamic laminate behavior by homogenization techniques. Wave Motion 16: 229-247

27. Santosa F, Vogelius M (1993) First-order corrections to homogenized eigenvalues of a periodic composite medium. SIAM J Appl Math 53(6):1636-1668

28. Smyshlyaev VP, Cherednichenko KD (2000) On rigorous derivation of strain gradient effects in the overall behaviour of periodic heterogeneous media. J Mech Phys Solids 48:1325-1357

29. Steinberg BZ, McCoy JJ (1994) Towards effective parameter theories using multiresolution decomposition. J Acoust Soc Am 96:1130-1143

30. Steinberg BZ, Mccoy JJ, Mirotznik M (2000) A multiresolution approach to homogenization and effective modal analysis of complex boundary value problems. SIAM J Appl Math 60(3):939-966

31. Zohdi TI, Wriggers P, Huet C (2001) A method of substructuring large-scale computational micromechanical problems. Comput Methods Appl Mech Eng 190(43):5639-5656 